\title{
Oxygen vacancies of YIG influence on the MPE in Au/YIG heterostructures
}

\author{
Xiao Liang ${ }^{1,2,3, a}$, Fei huang ${ }^{1,2,3}$,Chuangtang Wang ${ }^{1,2,3}$, Yupeng Zhu ${ }^{1,2,3}$,Yan \\ Zhang $^{1,2,3}$, Yue $\mathrm{Li}^{1,2,3}$ and Lei $\mathrm{Bi}^{1,2,3, \mathrm{~b}}$ \\ ${ }^{1}$ University of Electronic Science and Technology of China, Chengdu 610054, China \\ ${ }^{2}$ National Engineering Research Center of Electromagnetic Radiation Control Materials, University \\ of Electronic Science and Technology of China, Chengdu 610054,China \\ ${ }^{3}$ Key Laboratory of Multi-spectral Absorbing Materials and Structures, University of Electronic \\ Science and Technology of China, Ministry of Education, Chengdu 610054, China \\ a631842730@qq.com b bilei@uestc.edu.cn
}

Keywords: Oxygen vacancies, Magnetic proximity effect, Au/YIG heterostructures.

\begin{abstract}
The oxygen vacancies of $\mathrm{Y}_{3} \mathrm{Fe}_{5} \mathrm{O}_{12}(\mathrm{YIG})$ influence on the magnetic proximity effect (MPE) in Au/YIG heterostructures has been studied by first principles calculations based on the density functional theory (DFT). The results show that one oxygen vacancies can reduce the two neighbor $\mathrm{Fe}^{3+}$ to $\mathrm{Fe}^{2+}$ and MPE in Au/YIG is strongly depended on the valence states of Fe ion. The induced magnetism in $\mathrm{Au}$ atoms is found to increase with the increasing concentration of $\mathrm{Fe}^{2+}$. In oxygen vacancy free case $\left(\mathrm{Fe}^{3+}\right.$ state), weak electron states hybridization of $\mathrm{Fe}(3 \mathrm{~d})$ and $\mathrm{Au}(5 \mathrm{~d})$ is found form the projected electron density of states distribution, while after introduce the oxygen vacancy $\left(\mathrm{Fe}^{2+}\right.$ state), the states of $\mathrm{Fe}(3 \mathrm{~d})$ are generally move to higher energy states and hybridize with the $\mathrm{Au}(5 \mathrm{~d})$ states. Moreover, more states of $\mathrm{Fe}^{2+}$ at tetrahedral sites are hybridize with the $\mathrm{Au}(5 \mathrm{~d})$ states compare with that of $\mathrm{Fe}^{2+}$ at octahedral sites, therefore a net magnetic moment induced $\mathrm{Au}$ atoms is found and the direction of spin polarization of Au atoms is paralleled to Fe ion at tetrahedral sites.
\end{abstract}

\section{Introduction}

In recent years there has been a very strong interest in the magnetic proximity effect (MPE) in $\mathrm{Au}(\mathrm{Pt}) / \mathrm{Y}_{3} \mathrm{Fe}_{5} \mathrm{O}_{12}$ (YIG) heterostructures, which directly affects the interpretations of a number of recent important experimental observations of spintronics in the $\mathrm{Au}(\mathrm{Pt}) / \mathrm{YIG}$ system [1-3]. The magnetic proximity effect is a phenomenon of ferromagnetic ordering of spins within several atomic layers of a noble metal (NM) film in close proximity to a ferromagnetic material [4-6]. In previous both theoretical and experimental study, the MPE in Au/YIG is insignificant which is an order of magnitude weaker than that in Pt/YIG [7-9]. However, Francesco Pineider et al. found that the MPE in $\mathrm{Au} / \mathrm{FeO}$ is much stronger than that in $\mathrm{Au} / \mathrm{Fe}_{2} \mathrm{O}_{3}$ [10], which indicated MPE maybe existed in the $\mathrm{Au}$ /Iron oxide heterostructures while the $\mathrm{Fe}^{3+}$ is reduced to $\mathrm{Fe}^{2+}$. In each YIG unit cell, one oxygen vacancy can reduce two $\mathrm{Fe}^{3+}$ to $\mathrm{Fe}^{2+}$ at octahedral and tetrahedral sites, respectively [11]. With the aim of providing a theoretical prediction the MPE in Au/YIG bi-layered system while oxygen vacancies induced in YIG layer, first-principles calculations were carried out. The calculations are based on density functional theory (DFT). The results show that with the increasing of oxygen vacancies concentration, the MPE in Au/YIG become stronger and stronger because of the $\mathrm{Au}(5 \mathrm{~d})-\mathrm{Fe}^{2+}(3 \mathrm{~d})$ states hybridization.

\section{Calculation method}

In this paper, we applied the first-principles calculation method based on the density functional theory (DFT) to study oxygen vacancies of YIG influence on the MPE in Au/YIG heterostructures. The Perdew-Burke-Ernzerhof (PBE) exchange-correlation function [12] for the 
generalized-gradient-approximation (GGA) and a plane-wave basis set within the framework of the projector augmented wave (PAW) method were employed [13]. For the sampling of the Brillouin zone, we employed the Monkhorst-Pack scheme and used $3 \times 3 \times 1$ k-point grids for calculations. The $\mathrm{DFT}+\mathrm{U}$ formalism developed by Dudarev et al was used to account for the strong on-site Coulomb repulsion for the localized Fe (3d) states. The value of " $U$ " used for Fe (3d) states in our calculations is $4.3 \mathrm{eV}$. This value has been widely used in YIG with slight Ce doping and other systems containing $\mathrm{Fe}^{3+}$ cations [14]. To model the Au/YIG interfaces, we constructed a superlattice structure with a slab of YIG(100) of about 6 angstrom thick along with four Au(100) atomic layers on top. A 15 angstrom thick vacuum layer was inserted to avoid the interaction between the two cells as shown in Figs. 1(a) and 1(b). During the relaxation process, the in-plane lattice constant was fixed at the experimental value of the bulk YIG material, with the dimensions of $12.376 \times 12.376 \AA^{2}$. Due to the lattice misfit, the Pt layers were under the biaxial tensile strains of 1.1\%. All atoms were fully relaxed until the calculated force on each atom is smaller than $0.02 \mathrm{eV} / \AA$.

\section{Results and Discussions}

In a unit cell of YIG, the octahedral Fe ( $\left.\mathrm{Fe}^{\mathrm{oct}}\right)$ arranges in 8 body-centered cubic (BCC) subunit cells; the yttrium and tetrahedral $\mathrm{Fe}\left(\mathrm{Fe}^{\text {tet }}\right)$ are located on each surface of the 8 subunit cells, and the oxygen ions surrounds the Y and Fe ions with dodecahedral, octahedral, and tetrahedral coordination respectively. Due to the super exchange effect, the spin polarization directions of $\mathrm{Fe}^{\text {tet }}$ and $\mathrm{Fe}^{\mathrm{oct}}$ are opposite. For convenient, we denote spin up and spin down directions for the $\mathrm{Fe}^{\text {tet }}$ and $\mathrm{Fe}^{\text {oct }}$ ion, respectively. The calculations considered 6- $\AA$-thick YIG slabs because the induced moments converge at a YIG slab thickness of $6 \AA$ when the thickness was increased from $3 \AA$ to $9 \AA$. To investigate the effects of the oxygen vacancies of YIG on the MPE in Au/YIG, one, two, and four oxygen vacancies are induced at the interface of Au/YIG structure, respectively, and a 15 angstrom thick vacuum layer was inserted to avoid the interaction between the two cells as shown in fig. 1(a)-(c). Fig. 1(e) shows the calculated moments ( $\mu_{B} / A u$ atom) in each Au layer. One can see that by inducing oxygen vacancies (VO) at $\mathrm{Au} / \mathrm{YIG}$ interface, the magnetic moments of first Au atomic layer significantly enhanced comparing with the defect free case and then rapidly decreased. The average induced magnetic moment over all Au atoms, which indicates the strength of the MPE, are $0.006 \mu_{\mathrm{B}}$ /atom, $0.008 \mu_{\mathrm{B}} /$ atom, $0.015 \mu_{\mathrm{B}}$ /atom for $1 \mathrm{VO}, 2 \mathrm{VO}$, and $4 \mathrm{VO}$ cases. For comparing, the $\mathrm{Pt} / \mathrm{YIG}$ system without defects is also calculated, and the average induced magnetic moment over all $\mathrm{Pt}$ atoms is 0.070, which is still much larger than Au/YIG(4VO) system. Fig.1 (d) shows the structure of $\mathrm{Au} / \mathrm{YIG}(2 \mathrm{VO})$ system, where the 2VO located at the different atomic layers in the YIG. From the calculated moments in Au as shown in fig.1 (f), it is found that the induced magnetic moments of $\mathrm{Au}$ in the $\mathrm{Au} / \mathrm{YIG}(2 \mathrm{VO})$ - I system are lager than that in Au/YIG(2VO)- II system, which indicates the oxygen vacancies with different distance from the interface have unequal effect on the MPE, and the effect becomes weaker while the oxygen vacancy gradually away from the interface. Table 1 lists out the quantitative results of the magnetic moments induced in different systems. One can see by introducing oxygen vacancies to the YIG lattice, the magnetic moments of Au can enhance several times.

Table 1. In different Au/YIG system as shown in fig.1(a-d), the concentration of $\mathrm{Fe}^{2+}$ in YIG layer, the average magnetic moments in $1^{\text {st }}-4^{\text {th }} \mathrm{Au}(\mathrm{Pt})$ atomic layers $\left(\mathrm{M}_{1}-\mathrm{M}_{4}\right)$ and over all $\mathrm{Au}$ atoms $\left(\mathrm{M}_{0}\right)$ are listed. $\Delta \mathrm{M}$ is the percent variations in $\mathrm{M} 0$ comparing with the oxygen vacancy free case.

\begin{tabular}{cccccccc}
\hline System & $\mathrm{Fe}^{2+} \%$ & $\mathrm{M}_{1}$ & $\mathrm{M}_{2}$ & $\mathrm{M}_{3}$ & $\mathrm{M}_{4}$ & $\mathrm{M}_{0}$ & $\Delta \mathrm{M}$ \\
\hline $\mathrm{Au} / \mathrm{YIG}$ & $0 \%$ & 0.004 & 0.002 & 0.002 & 0.000 & 0.002 & $\ldots$ \\
$\mathrm{Au} / \mathrm{YIG}(1 \mathrm{VO})$ & $7 \%$ & 0.017 & 0.006 & 0.002 & -0.001 & 0.006 & $200 \%$ \\
$\mathrm{Au} / \mathrm{YIG}(2 \mathrm{VO}-\mathrm{I})$ & $14 \%$ & 0.022 & 0.009 & 0.002 & -0.002 & 0.008 & $300 \%$ \\
$\mathrm{Au} / \mathrm{YIG}(2 \mathrm{VO}-\mathrm{II})$ & $14 \%$ & 0.018 & 0.003 & 0.000 & -0.001 & 0.005 & $250 \%$ \\
$\mathrm{Au} / \mathrm{YIG}(4 \mathrm{VO})$ & $28 \%$ & 0.031 & 0.010 & 0.013 & 0.008 & 0.015 & $650 \%$ \\
$\mathrm{Pt} / \mathrm{YIG}$ & $0 \%$ & 0.041 & 0.065 & 0.091 & 0.081 & 0.070 & $\ldots$ \\
\hline
\end{tabular}




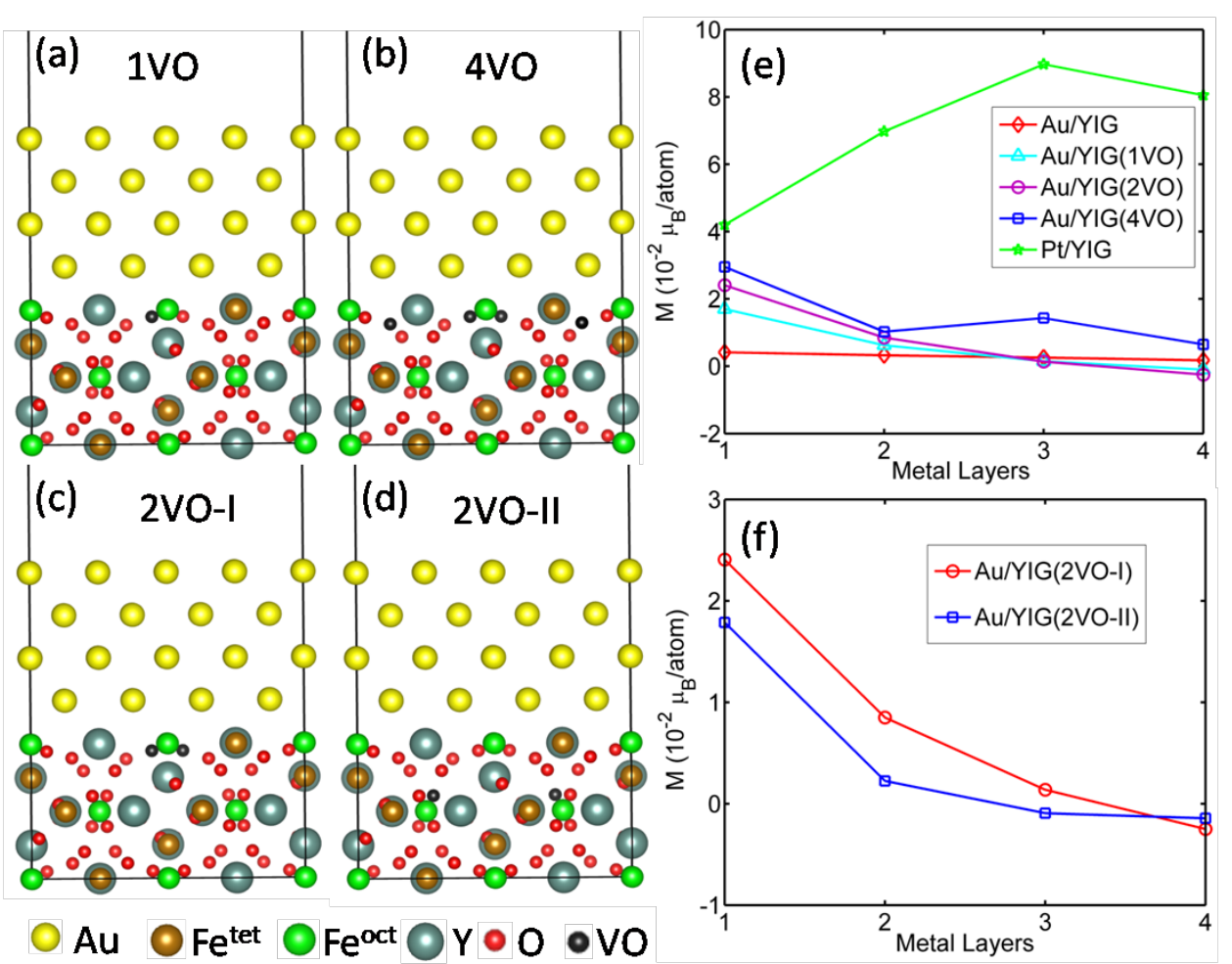

Figure 1. Side view of the calculated crystalline structures of Au(100)/YIG(100) in different oxygen vacancies induced cases: one (a), two(c) and four(b) oxygen vacancies located at the interface; two oxygen vacancies located at the deeper layer of YIG (d). (e)(f) show the induced magnetic moments of each layer $\mathrm{Au}(\mathrm{Pt})$ in different oxygen vacancies doped cases.

To reveal the origin of the oxygen vacancies increasing the magnetic moments of $\mathrm{Au}$ in $\mathrm{Au} / \mathrm{YIG}$ system, the projected density of states (PDOS) is studied. Fig. 2(a) and (b) show the PDOS of the interface $\mathrm{Au}, \mathrm{Fe}^{\text {tet }}$ and $\mathrm{Fe}^{\text {oct }}$ ion in $\mathrm{Au} / \mathrm{YIG}$ without and with oxygen vacancies cases, respectively. The DOS peaks of $\mathrm{Fe}^{\text {tet }}$ and $\mathrm{Fe}^{\text {oct }}$ neighbor to the oxygen ion below the Fermi leave are both located above $-7 \mathrm{eV}$, which is agree with other calculation results of PDOS in bulk YIG [11]. On the contrary, the locations of peaks of $\mathrm{Fe}^{\text {tet }}$ and $\mathrm{Fe}^{\text {oct }}$ the oxygen vacancy moved $1.56 \mathrm{eV}$ and $1.03 \mathrm{eV}$ near to the Fermi level, and more states of $\mathrm{Fe}^{\text {tet }}$ are hybridization with $\mathrm{Au} 5 \mathrm{~d}$ states comparing to that of $\mathrm{Fe}^{\text {oct }}$. Simultaneously, we can see an additional down spin and up spin peak are added for $\mathrm{Fe}^{\text {tet }}$ and $\mathrm{Fe}^{\text {oct }}$, which indicates the $\mathrm{Fe}^{3+}$ is reduced to $\mathrm{Fe}^{2+}$ due to the oxygen vacancy. Therefore, through electrons exchange effect between $\mathrm{Fe}^{2+}$ and $\mathrm{Au}$, the magnetic moment is induced in $\mathrm{Au}$ and the direction is along the spin polarization direction of $\mathrm{Fe}^{\mathrm{tet}}$. The different strength of the electron hybridization between Pt and Fe ions induces significantly different magnetic properties of Pt due to direct exchange, which leads to different MPE responses in Pt/YIG with different interface structures. 


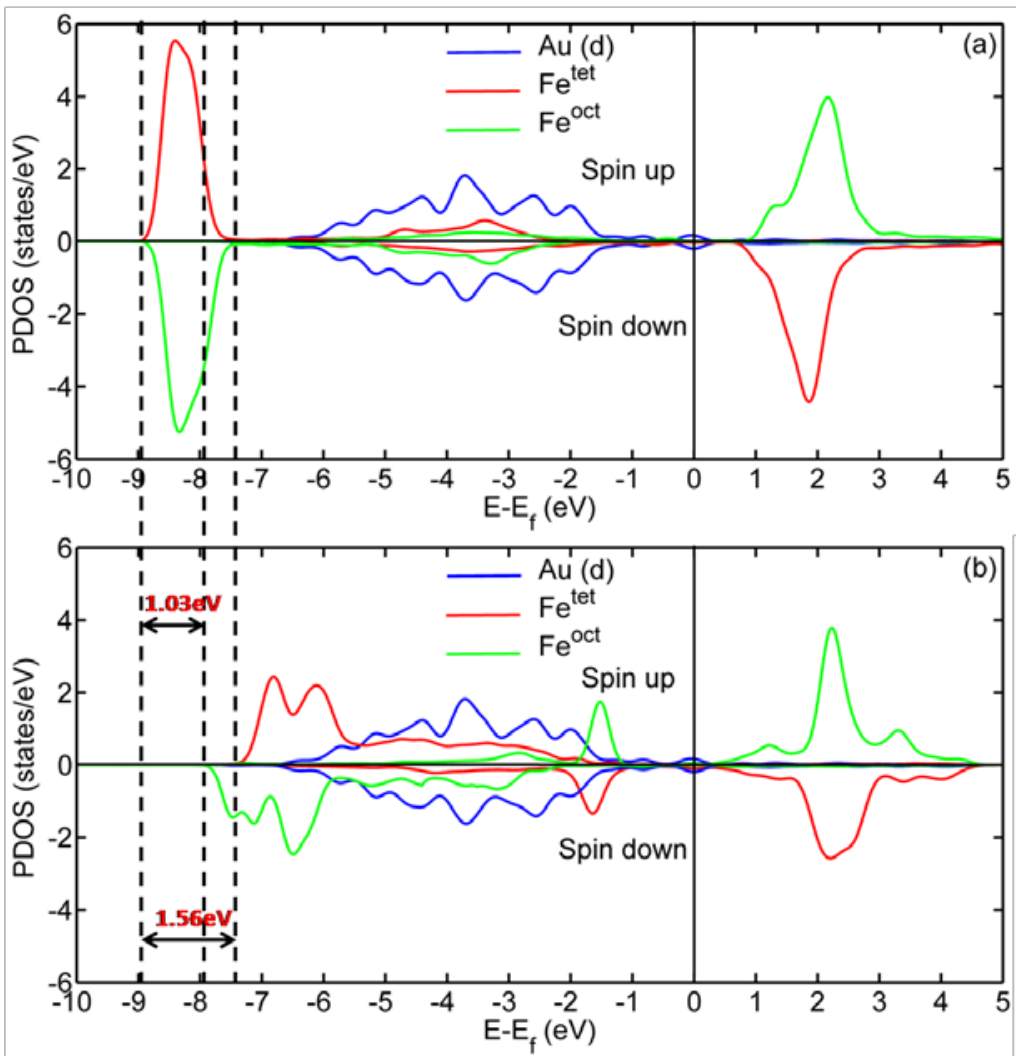

Figure 2. The projected density of states (PDOS) of the interface $\mathrm{Au}, \mathrm{Fe}^{\text {tet }}$ and $\mathrm{Fe}^{\mathrm{oct}}$ ion in $\mathrm{Au} / \mathrm{YIG}$ heterostructures without (a) and with (b) oxygen vacancies.

\section{Summary}

In summary, we studied the oxygen vacancies influence on the magnetic proximity effect of $\mathrm{Au} /$ YIG bi-layered heterostructures using first principles calculations. The results show with the increasing concentration of oxygen vacancies at the Au/YIG interface, the MPE in Au/YIG heterostructure become stronger and stronger. The physical origin of this effect can be explained by the projected electron density of states distribution, which can be described as the following: by introducing the oxygen vacancy, the $\mathrm{Fe}^{3+}$ in YIG is reduced to $\mathrm{Fe}^{2+}$, then the $\mathrm{Fe}(3 \mathrm{~d})$ states move to higher energy level and hybridize with $\mathrm{Au}(5 \mathrm{~d})$ states. Through the electron exchange effect, magnetism is induced to Au atoms. In addition, we also found he magnetism of Au atom become weaker while the oxygen vacancies are moved further layer from the Au/YIG interface.

\section{Acknowledgments}

The authors are grateful to the support of National Natural Science Foundation of China (61475031, 51302027, 51522204), the Fundamental Research Funds for the Central Universities (ZYGX2013J028, ZYGX2014Z001), and the Science Foundation for Youths of Sichuan Province (2015JQO014).

\section{References}

[1]. Y. M. Lu, Y. Choi, C. M. Ortega, et al., Pt Magnetic Polarization on $\mathrm{Y}_{3} \mathrm{Fe}_{5} \mathrm{O}_{12}$ and Magnetotransport Characteristics, Phys. Rev. Lett. 110 (2013) 147207(1) -147207(5).

[2]. H. Nakayama, M. Althammer, Y. T. Chen, et al., Spin Hall Magnetoresistance Induced by a Nonequilibrium Proximity Effect. Phys. Rev. Lett. 110 (2013) 206601(1)-206601(5). 
[3]. T. Lin, C. Tang, H. M. Alyahayaei, et al., Experimental Investigation of the Nature of the Magnetoresistance Effects in Pd-YIG Hybrid Structures. Phys. Rev. Lett. 110 (2014) 372030(1)- 372030(5).

[4]. Y. Y. Sun, H. Chang, M. Kabatek, et al., Damping in Yttrium Iron Garnet Nanoscale Films Capped by Platinum, Phys. Rev. Lett. 111 (2013) 106601(1) -106601(5).

[5]. B. Heinrich, Y. Tserkovnyak, G. Woltersdorf, et al., Dynamic Exchange Coupling in Magnetic Bilayers. Phys. Rev. Lett. 90 (2003) 187601(1)-187601(4).

[6]. S. Y. Huang, X. Fan, D. Qu, et al. Transport Magnetic Proximity Effects in Platinum, Phys. Rev. Lett. 109 (2012) 107204(1)-107204(5).

[7]. D. Qu, S. Y. Huang, J. Hu, et al., Intrinsic Spin Seebeck Effect in Au/YIG, Phys. Rev. Lett. 110 (2013) 067206(1)- 067206(5).

[8]. B. F. Miao, S. Y. Huang, D. Qu, et al., Physical Origins of the New Magnetoresistance in Pt/YIG, Phys. Rev. Lett. 112 (2014) 236601(1)- 236601(5).

[9]. X. Liang, Y. Zhu, B. Peng, et al., Influence of Interface Structure on Magnetic Proximity Effect in $\mathrm{Pt} / \mathrm{Y}_{3} \mathrm{Fe}_{5} \mathrm{O}_{12}$ Heterostructures. ACS Appl. Mater. Interface. 8 (2016) 8175-8183.

[10]. F. Pineider, C. J. Fernández, V. Videtta, et al., Spin-Polarization Transfer in Colloidal Magnetic-Plasmonic Au/Iron Oxide Hetero-nanocrystals. ACS Nono. 7 (2013) 857-866.

[11]. X. Liang, J. Xie, L. Deng, et al., First Principles Calculation on the Magnetic, Optical Properties and Oxygen Vacancy Effect of $\mathrm{Ce}_{\mathrm{x}} \mathrm{Y}_{3-\mathrm{x}} \mathrm{Fe}_{5} \mathrm{O}_{12}$. Appl. Phys. Lett. 106 (2015) 052401(1)- 052401(5).

[12]. J. P. Perdew, K. Burke, and M. Ernzerhof, Generalized Gradient Approximation Made Simple. Phys. Rev. Lett. 77 (1996) 3865-3868.

[13]. G. Kresse, and D. Joubert, From Ultrasoft Pseudopotentials to the Projector Augmented-Wave Method. Phys. Rev. B 59 (1999) 1758-1775.

[14]. X. Guo, A. H. Tavakoli, S. Sutton, et al. Cerium Substitution in Yttrium Iron Garnet: Valence State, Structure, and Energetics. Chem. Mater. 26 (2013) 1133-1143. 